\title{
Experimental methods to characterise complex fluids
}

\author{
Volfango Bertola ${ }^{*}$ Teodor Burghelea ${ }^{\dagger}$ \\ * School of Engineering, University of Liverpool, Liverpool, United Kingdom \\ † Université de Nantes, Nantes Atlantique Universités,Laboratoire de Thermique \\ et Energie de Nantes, UMR 6607, La Chantrerie, Rue Christian Pauc, B.P. \\ 50609, F-44306 Nantes Cedex 3, France
}

Abstract the abstract goes here

\section{Contents}

\section{Contents}

1 Rheological characterisation of complex fluids 3

1.1 Fundamentals of shear rheology . . . . . . . . . . . . 3

1.2 Fundamentals of extensional rheology . . . . . . . . . . . 12

\section{Bibliography}

\section{List of Figures}

1 Schematic representation of the capillary rheometer. . . . . . . . 5

2 (a) Schematic representation of the Taylor-Couette setup. (b) Choice of the cylindrical coordinates. . . . . . . . . . . . 7

3 (a) Schematic representation of the cone-plate setup. (b) Choice of the spherical coordinates. . . . . . . . . . . . . . 10

4 (a) Schematic representation of the parallel plate setup. (b) Choice of the cylindrical coordinates. . . . . . . . . . . . . . 11

5 Steady elongational flow field $(b=0) . \ldots \ldots \ldots$

6 Deformation of a cube of unitary volume (a) during the time interval $\Delta t$ by three types of shear free flow: (b) uniaxial extension $(b=0, \dot{\epsilon}>0)$, (c) biaxial extension $(b=0, \dot{\epsilon}<0)$, (d) planar elogation $(b=1)$. 
$7 \quad$ Schematic represenation of the capillary breakup device: (a) initial fluid meniscus. (b) fluid meniscus during the capillary thinning process. . . . . . . . . . . . . . 17

8 Schematic representation of the Meissner extensional rheometer. 20

9 Schematic representation of the Münstedt extensional rheometer according to Ref. Münstedt (1979) (not in scale): (S) - sample, (OB) - oil bath, $\left(P_{1}\right)$ - top plate, $\left(P_{2}\right)$ - bottom plate. . . . . . 21

10 Schematic representation of the filament stretching rheometer according to Ref. Tirtaatmadja and Sridhar (1993) (not in scale). 23

11 Schematic representation of the Sentmanat extensional fixture: (a) front view (b) top view. . . . . . . . . . . . . . 25 


\section{Rheological characterisation of complex fluids}

Rheology is one of the very few scientific disciplines whose emergence can be precisely dated: April 29, 1929, Bingham (cca 1945). The term "Rheology" has been coined by Dr. Eugene Bingham, professor at the Lafayette College following a suggestion of his colleague Dr. Markus Rainer and was inspired by an aphorism of Simplicius (often but incorrectly attributed to Heraclitus) "pantha rhei" - "everything flows". The main scope of rheology is the study of deformation and flow of fluids and soft solids subjected to a varying an external stress. The rheology is equally concerned with establishing a correlation between the molecular structure of the materials and their flow properties which is of paramount importance during polymer processing operations.

\subsection{Fundamentals of shear rheology}

In order to relate the stresses and the deformations measured by a rheometer, we refer to the Navier-Stokes equation:

$$
\rho \frac{d \mathbf{v}}{d t}=\nabla \cdot(-p \delta+\sigma)+\rho \mathbf{g}
$$

where $\sigma$ is the stress tensor, $p$ is the pressure field, $\delta$ is the unitary tensor and the body force $\mathbf{g}$ is just acceleration of the gravity. We note that the inertial term $\mathbf{v} \nabla \mathbf{v}$ has been omitted in the equation of motion Eq. 1.

For a steady flow $\frac{d \mathbf{v}}{d t}=0$ and the momentum equation reduces to:

$$
\nabla \cdot(-p \delta+\sigma)+\rho \mathbf{g}=0
$$

If one projects Eq. 2 onto a Cartesian system of coordinates one obtains:

$$
\begin{gathered}
\frac{\partial \sigma_{x x}}{\partial x}+\frac{\partial \sigma_{y x}}{\partial y}+\frac{\partial \sigma_{z x}}{\partial z}=\frac{\partial p}{\partial x}-\rho g_{x} \\
\frac{\partial \sigma_{x y}}{\partial x}+\frac{\partial \sigma_{y y}}{\partial y}+\frac{\partial \sigma_{y z}}{\partial z}=\frac{\partial p}{\partial y}-\rho g_{y} \\
\frac{\partial \sigma_{x z}}{\partial x}+\frac{\partial \sigma_{y z}}{\partial y}+\frac{\partial \sigma_{z z}}{\partial z}=\frac{\partial p}{\partial z}-\rho g_{z}
\end{gathered}
$$

If one projects Eq. 2 onto a cylindrical system of coordinates one obtains: 


$$
\begin{array}{r}
\frac{\partial \sigma_{r r}}{\partial r}+\frac{1}{r} \frac{\partial \sigma_{r \phi}}{\partial \phi}+\frac{\partial \sigma_{r z}}{\partial z}+\frac{\sigma_{r r}-\sigma_{\phi \phi}}{r}=\frac{\partial p}{\partial r}-\rho g_{r} \\
\frac{\partial \sigma_{r \phi}}{\partial r}+\frac{1}{r} \frac{\partial \sigma_{\phi \phi}}{\partial \phi}+\frac{\partial \sigma_{\phi z}}{\partial z}+\frac{2 \sigma_{r \phi}}{r}=\frac{1}{r} \frac{\partial p}{\partial \theta}-\rho g_{\phi} \\
\frac{\partial \sigma_{r z}}{\partial r}+\frac{1}{r} \frac{\partial \sigma_{z \phi}}{\partial \phi}+\frac{\partial \sigma_{z z}}{\partial z}+\frac{\sigma_{r z}}{r}=\frac{\partial p}{\partial z}-\rho g_{z}
\end{array}
$$

If one projects Eq. 2 onto a spherical system of coordinates one obtains:

$$
\begin{array}{r}
\frac{\partial \sigma_{r r}}{\partial r}+\frac{1}{r} \frac{\partial \sigma_{r \theta}}{\partial \theta}+\frac{1}{r \sin \theta} \frac{\partial \sigma_{r \phi}}{\partial \phi}+\frac{2 \sigma_{r r}-\sigma_{\theta \theta}-\sigma_{\phi \phi}+\sigma_{\theta \theta} \cot \theta}{r}=\frac{\partial p}{\partial r}-\rho g_{r} \\
\frac{\partial \sigma_{r \phi}}{\partial r}+\frac{1}{r} \frac{\partial \sigma_{\theta \phi}}{\partial \theta}+\frac{1}{r \sin \theta} \frac{\partial \sigma_{\phi \phi}}{\partial \phi}+\frac{2 \sigma_{r \phi}-\sigma_{\phi r}+\left(\sigma_{\theta \phi}+\sigma_{\phi \theta}\right) \cot \theta}{r}=\frac{1}{r} \frac{\partial p}{\partial \theta}-\rho g_{\phi} \\
\frac{\partial \sigma_{r \theta}}{\partial r}+\frac{1}{r} \frac{\partial \sigma_{\theta \theta}}{\partial \theta}+\frac{1}{r \sin \theta} \frac{\partial \sigma_{\theta \phi}}{\partial \phi}+\frac{\sigma_{\theta r}+2 \sigma_{r \theta}+\left(\sigma_{\theta \theta}-\sigma_{\phi \phi}\right) \cot \theta}{r}=\frac{1}{r \sin \theta} \frac{\partial p}{\partial \phi}-\rho g_{\theta}
\end{array}
$$

Capillary rheometry During the early days of the rheology, one of the most commonly used rheometric systems was the capillary rheometer schematically illustrated in Fig. 1. The fluid to be tested initially contained in a reservoir flows through a capillary of radius $R$ and length $L$ in conditions of a controlled applied pressure $P$ that can be supplied either by gravity (in the case of low viscosity fluids) or by the motion of a piston inside the reservoir (in the case of highly viscous fluids, e.g. molten polymers). The viscosity of the fluid may be inferred by simultaneous measurements of the driving pressure $P$ and of the flow rate $Q$ through the capillary if the following conditions are satisfied:

1. The flow is isothermal.

2. The flow is fully developed, linear and laminar.

3. The fluid is incompressible with a viscosity independent on the driving pressure.

4. There exists no slip at the wall, $\left.v_{z}\right|_{r=R}=0$.

The first condition above indicates that, in order to solve such flow problem and assess the viscosity, one needs to refer solely to the momentum conservation equation as there exists no transfer of heat. The linear and laminar nature of the flow translates into the absence of any radial and azimuthal flow component, $v_{r}=v_{\theta}=0$. With these remarks, the Navier-Stokes equation reduces to:

$$
-\frac{\partial p}{\partial z}+\frac{1}{r} \frac{\partial r \sigma_{r z}}{\partial r}=0
$$




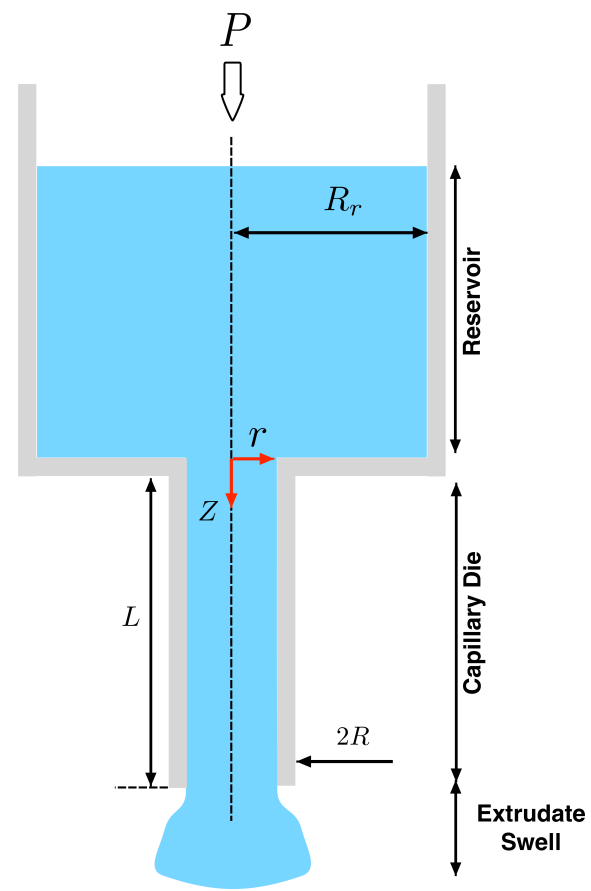

Figure 1. Schematic representation of the capillary rheometer.

Using the assumption that the flow is fully developed, the term $\frac{\partial p}{\partial z}$ is constant along the capillary tube and Eq. 6 may be integrated to:

$$
\sigma_{r z}=\frac{r}{2} \frac{P_{c}}{L}
$$

where $P_{c}$ is the pressure drop along the capillary tube. The stress at the wall of the die is $\sigma_{w}=\left.\sigma_{r z}\right|_{r=R}=\frac{R}{2} \frac{P_{c}}{L}$. To compute the shear viscosity one needs the rate of shear in the capillary $\dot{\gamma}=\frac{d v_{z}}{d r}$. This may be obtained from the measured flow rate $Q$ by noting that its relationship with the axial flow speed:

$$
Q=2 \pi \int_{0}^{R} r v_{z}(r) d r
$$

By integrating Eq. 8 and using the no slip boundary conditions one may readily show: 


$$
\frac{Q \sigma_{w}^{3}}{\pi R^{3}}=-\int_{0}^{\sigma_{w}} \sigma_{r z}^{2}\left(\frac{d v_{z}}{d r}\right) d \sigma_{r z}
$$

Finally, by differentiating the equation above with respect to $\sigma_{w}$ according to the fundamental theorem of calculus and re-arranging the terms one obtains the Weissenberg-Rabinowitsch equation:

$$
-\frac{d v_{z}}{d r} \mid \sigma_{w}=\dot{\gamma}_{w}=\frac{1}{4} \dot{\gamma}_{a w}\left[3+\frac{d \ln Q}{d \ln \sigma_{w}}\right]
$$

with the apparent shear rate given by $\dot{\gamma}_{a w}=\frac{4 Q}{\pi R^{3}}$.

By measuring the pressure drop $P_{c}$ and the flow rate and if $n=\frac{d \ln Q}{d \ln \sigma_{w}}$ can be reliably computed via numerical differentiation of the data, one can compute the viscosity:

$$
\eta=\frac{\sigma_{w}}{\dot{\gamma}_{w}}=\frac{\pi R^{4} P_{c}}{2 Q L}\left(\frac{n}{3 n+1}\right)
$$

We note here that, in the case of a power law fluid, $n$ is simply the power law index. The capillary rheometer has some practical advantages. It is relatively easy to use and provides accurate steady state viscosity measurements. However, entry corrections require a more extensive data analysis procedure, Macosko (1994).

Concentric cylinders rheometry The first operational rotational rheometer was the device built by Maurice Couette, Couette (1880). Couette used a system of concentric cylinders containing within their gap the material to be tested, Fig. 2(a). In the original prototype of Couette, the outer cylinder of radius $r_{i}$ was rotating at a constant angular speed and the inner cylinder of radius $r_{o}$ was suspended by a torsion wire. The torque acting on the inner cylinder was assessed by measuring the angular deflection using a mirror rigidly attached on the torsional wire. The modern Couette devices use similar operating systems with the difference that, for practical reasons, the inner cylinder is set in rotation by attaching it to the shaft of a rheometer while the outer one is static, Fig. 2(a). To describe the flow kinematics in a Couette device we use the cylindrical coordinates illustrated in Fig. 2(b). One can readily show that the shear rate in a Couette device is $\dot{\gamma}=r \frac{d \Omega}{d r}$. Within the narrow gap approximation, $\frac{r_{o}-r_{i}}{r} \ll 1$, the following approximation can be made:

$$
\frac{d \Omega}{d r} \approx \frac{\Delta \Omega}{\Delta r}=\frac{\Omega}{r_{o}-r_{i}}
$$




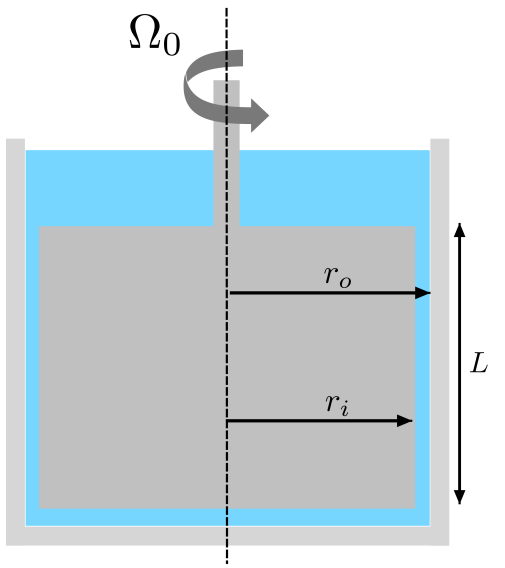

(a)

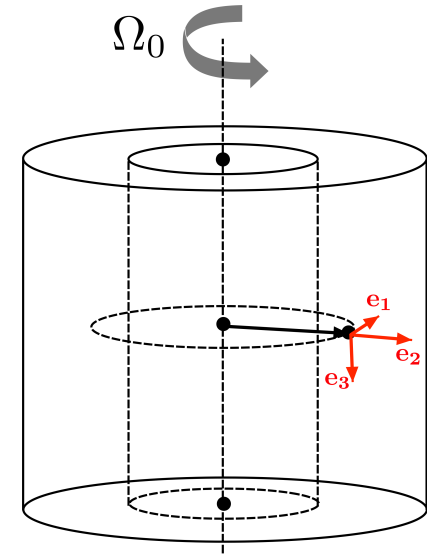

(b)

Figure 2. (a) Schematic representation of the Taylor-Couette setup. (b) Choice of the cylindrical coordinates.

with $\Delta r=r_{o}-r_{i}$ and $\Delta \Omega=\Omega\left(r_{o}\right)-\Omega\left(r_{i}\right)=\Omega_{0}$. With this approximation, the shear rate becomes:

$$
\dot{\gamma} \approx r \frac{\Omega_{0}}{\Delta r} \approx \frac{r_{i}+r_{o}}{2} \frac{\Omega_{0}}{\Delta r}=\frac{r_{a} v}{\Delta r} \Omega_{0}
$$

with the average radius $r_{a v}=\frac{r_{i}+r_{o}}{2}$. It can be shown that yet a better approximation for the shear rate is:

$$
\dot{\gamma}(r) \approx \frac{\Omega_{0}}{r^{2}} \frac{r_{i}^{2} r_{o}^{2}}{r_{a v} \Delta r}
$$

Due to symmetry considerations, a number of terms in the equation of motion will vanish: $\sigma_{\phi z}=\sigma_{z \phi}=\sigma_{r z}=\sigma_{z r}=0$. Moreover, in the laminar axisymmetric case, all partial derivatives with respect to the polar angle are zero, $\frac{\partial}{\partial \phi}=0$. The equations of motion Eqs.4 reduce to:

$$
\begin{aligned}
\frac{\partial \sigma_{r r}}{\partial r}-\frac{\left(\sigma_{\phi \phi}-\sigma_{r r}\right)}{r}=0 \Longrightarrow \frac{\partial \sigma_{r r}}{\partial r}-\frac{N_{1}}{r} & =0 \\
\frac{\partial \sigma_{r \phi}}{\partial r}-2 \frac{\sigma_{r \phi}}{r} & =0 \\
\frac{\partial \sigma_{z z}}{\partial z} & =-\rho g
\end{aligned}
$$


From Eq. 16 one obtains $r \frac{\partial \sigma_{r r}}{\partial r}=N_{1}$. Within the narrow gap approximation, $\frac{\partial \sigma_{r r}}{\partial r} \approx \frac{\Delta \sigma_{r r}}{\Delta r}=\frac{\sigma_{r r}\left(r_{o}\right)-\sigma_{r r}\left(r_{i}\right)}{r_{o}-r_{i}}$. Finally, the first normal stress difference may be approximated by:

$$
N_{1} \approx \frac{\Delta \sigma_{r r}}{\Delta r} r_{a v}
$$

By integrating Eq. 17 one obtains $r^{2} \sigma_{r \phi}=C=$ constant. The torque acting on the rotating cylinder may be written $T=2 \pi r^{2} L \sigma_{r \phi}=2 \pi C L$. An important conclusion is that the torque does not depend on the position in the liquid. The shear viscosity can be readily computed:

$$
\eta=\frac{\sigma_{r \phi}}{\dot{\gamma}}=\frac{T}{4 \pi \Omega_{0} L} \frac{r_{o}^{2}-r_{i}^{2}}{r_{o}^{2} r_{i}^{2}}
$$

To conclude, the simultaneous measurements of the angular speed $\Omega_{0}$ and of the torque $T$ allow one to measure the viscosity (in the narrow gap limit).

Cone and plate rheometry In a cone-plate rheometric setup the material under investigation is confined within the gap between a cone with a large top angle (typically larger than $170^{\circ}$ ) and a flat plate, Fig. 3(a). Consequently, the angle between the cone and the plate is small, $\Delta \Theta \leq 5^{\circ}$. The top part of the cone is attached to a rotating shaft with the symmetry axis orthogonal to the plate and positioned at its centre. To avoid contact friction, the cone is truncated The shearing surfaces are co-axial conical surfaces with top angles ranging in between $\pi$ and $\pi-2 \Delta \Theta$. The natural coordinates one may use to describe the kinematics of the motion are the spherical coordinates, $3(\mathrm{~b})$.

The shear rate may be computed (for the details of the calculation the reader is referred to Refs. Macosko (1994); Bird et al. (1977)):

$$
\dot{\gamma}=\sin \theta \frac{d \Omega}{d \theta} \approx \frac{\Omega_{0}}{\Delta \Theta}
$$

According to Eq. 21, the shear rate is constant within the entire material under investigation which makes the cone-plate tool best suited for the measurements of viscosity and normal stress differences as a function of the applied shear. In the absence of hydrodynamic instabilities, the flow field has a single component, $v_{\phi}=v_{\phi}(r)$ and the equation of motion described by Eqs. 5 reduce to: 


$$
\begin{gathered}
\frac{\partial \sigma_{r r}}{\partial r}-\frac{N_{1}+2 N_{2}}{r}=-\rho g \cos \theta \\
\frac{1}{r} \frac{\partial \sigma_{\theta \theta}}{\partial \theta}-\frac{N_{1}}{r} \cot \theta=\rho g \sin \theta \\
\frac{1}{r} \frac{\partial \sigma_{\theta \phi}}{\partial \theta}+\frac{2 \sigma_{\theta \phi}}{r} \cot \theta=\rho g \sin \theta
\end{gathered}
$$

Bearing in mind that $\cot \theta \approx 0$, the integration of Eq. 24 leads to $\sigma_{\theta \phi}=$ constant $=C$. Consequently, the torque $T$ exerted on the top plate may be computed:

$$
T=\left.\int_{0}^{2 \pi} \int_{0}^{R} r^{2} \sigma_{\theta \phi}\right|_{\pi / 2} d r d \phi
$$

which leads to $\sigma_{\theta \phi}=\frac{3 T}{2 \pi R^{3}}$. The viscosity may be calculated as:

$$
\eta=\frac{\sigma_{\theta \phi}}{\dot{\gamma}}=\frac{3 T}{2 \pi \dot{\gamma} R^{3}} \approx \frac{3 T \Delta \Theta}{2 \pi R^{3} \Omega_{0}} .
$$

According to the equation above, the viscosity measured with a cone-plate geometry is proportional to the torque acting on the geometry and inverse proportional to its angular speed. Besides the homogeneity of the rate of shear within the entire volume of the sample under investigation, a second notable feature of the cone-plate rheometric setup is that, within the small angle approximation, the result given by Eq. 26 is independent of the constitutive equation of the material.

The normal stress differences $N_{1}=\sigma_{\phi \phi}-\sigma_{\theta \theta}, N_{2}=\sigma_{\theta \theta}-\sigma_{r r}$ can be measured by measuring the normal force and the pressure distribution along the bottom plate. Because $\cos \theta \approx 0$, Eq. 23 may be approximated ${ }^{1}$ by:

$$
r \frac{\partial \sigma_{r r}}{\partial r} \approx N_{1}+N_{2}
$$

As the shear rate $\dot{\gamma}$ is independent on the radial coordinate $r$ and $N_{2}$ is a steady material function depending only on $\dot{\gamma}, \frac{\partial N_{2}}{\partial r}=\frac{\sigma_{\theta \theta}}{\partial r}-\frac{\sigma_{r r}}{\partial r}=0$ or $\frac{\sigma_{\theta \theta}}{\partial r}=\frac{\sigma_{r r}}{\partial r}$. With these remarks, Eq. 26 becomes:

$$
\frac{\partial \sigma_{\theta \theta}}{\partial \ln r} \approx N_{1}+N_{2}
$$

The radial distribution of the stress $\sigma_{\theta \theta}$ may be measured using an array of pressure transducers mounted on the bottom plate at various radial positions $r$. If we can assume that the stress in the radial direction at the rim

\footnotetext{
${ }^{1}$ The relationship becomes exact only at the level of the plate, $\theta=\pi / 2$.
} 


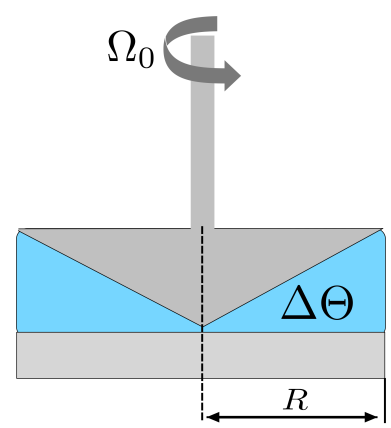

(a)

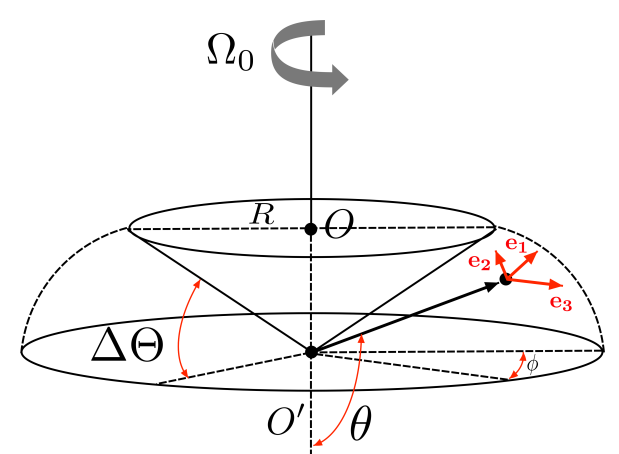

(b)

Figure 3. (a) Schematic representation of the cone-plate setup. (b) Choice of the spherical coordinates.

$\left.\sigma_{\theta \theta}\right|_{r=R}$ is balanced by the atmospheric pressure $p_{a}$ (i.e., the surface tension and/or other edge effects may be neglected), then $\sigma_{\theta \theta}(R)$ is just the second normal stress difference:

$$
N_{2}=\sigma_{\theta \theta}(R)-\sigma_{r r}(R)=\sigma_{\theta \theta}(R) .
$$

From a technical standpoint, it simpler to measure the normal force $F_{z}$ exerted on the bottom plate of the geometry:

$$
F_{z}=-p_{a} \pi R^{2}-\int_{0}^{2 \pi} \int_{0}^{R} \sigma_{\theta \theta}(r) d r d \phi .
$$

The integration of the equation above yields:

$$
F_{z}=\frac{1}{2} \pi R^{2} N_{1}
$$

To obtain the relationship above, one has assumed once more that the atmospheric pressure balances the pressure exerted on the free fluid meniscus. To conclude, by combining measurements of the thrust exerted on the bottom plate with measurements of the radial distribution of the pressure one can measure both normal force differerences.

Parallel plate rheometry Yet another rheometric setup commonly used for the rheological characterisation of fluids is the parallel plate torsional rheometer, Fig.4(a). The fluid to be investigated is confined in the gap 


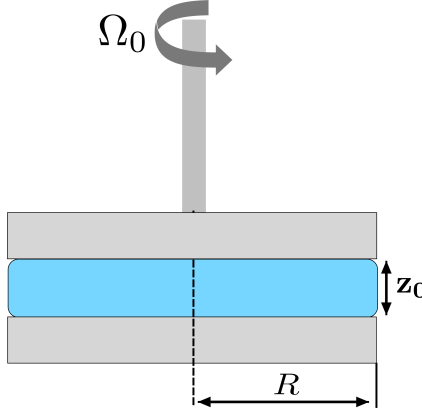

(a)

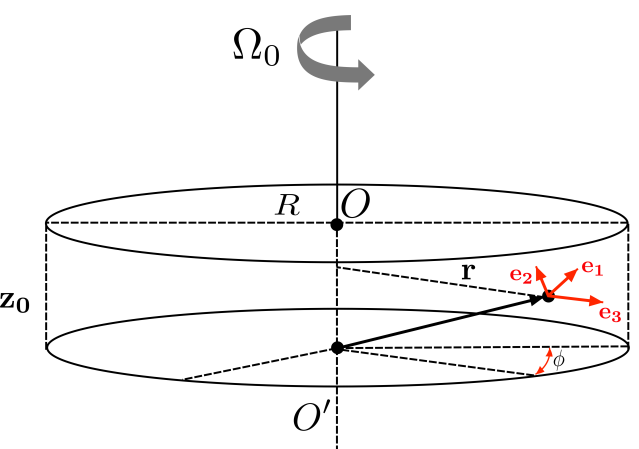

(b)

Figure 4. (a) Schematic representation of the parallel plate setup. (b) Choice of the cylindrical coordinates.

between two parallel plates separated by a finite distance $z_{0}$ ranging from hundreds of micro-meters to several milimeters. The bottom plate is fixed while the top plate rotates at a constant angular speed $\Omega_{0}$ around the common symmetry axis of the plates, Fig.4. In the absence of hydrodynamic instabilities, the trajectories of individual material elements are concentric circles. The natural coordinates one may use to describe the kinematics are the cylindrical coordinates detailed in Fig. 4(b). With this choice of coordinates, $v_{r}=v_{\phi}=v_{z}=0$.

The shear rate is may be expressed as:

$$
\dot{\gamma}=\lim _{\Delta z \rightarrow 0} \frac{r \Omega(z+\Delta z)-r \Omega(z)}{\Delta z}=r \frac{d \Omega}{d z}
$$

Unlike in the case of the cone-plate geometry, the rate of shear is not constant within the gap between the parallel plates. It increases linearly from $\dot{\gamma}=0$ along the symmetry axis of the device to $\dot{\gamma}(R)=\frac{\Omega_{0}}{z_{0}}$ at the rim. This is a significant drawback of the plate-plate system as it can not be used for materials with properties that depend strongly on the shear rate which is the case for a large number of materials that exhibit shear thinning rheological properties. 
The equations of motion may be written:

$$
\begin{array}{r}
\frac{\partial \sigma_{r r}}{\partial r}=\frac{N_{1}+N_{2}}{r} \\
\frac{\partial \sigma_{\phi z}}{\partial z}=0 \\
\frac{\partial \sigma_{z z}}{\partial z}=\rho g
\end{array}
$$

From Eq. 35 it can be shown:

$$
\begin{array}{r}
v_{\theta}(r, z)=\frac{r \Omega_{0} z}{z_{0}} \\
\dot{\gamma}(r)=r \frac{d \Omega}{d z}=r \frac{\Omega_{0}}{z_{0}}
\end{array}
$$

The relationship between the shear stress $\sigma_{\theta z}$ and the torque $T$ acting on the top plate is:

$$
\sigma_{\theta z}\left(\dot{\gamma}_{R}\right)=\frac{T}{2 \pi R^{3}}\left[3+\frac{d \ln T}{d \ln \dot{\gamma}_{R}}\right]
$$

where the rim shear rate is $\dot{\gamma}_{R}=R \frac{\Omega_{0}}{z_{0}}$. The difference between the first and the second normal stress differences is:

$$
N_{1}\left(\dot{\gamma}_{R}\right)-N_{2}\left(\dot{\gamma}_{R}\right)=\frac{F_{z}}{\pi R^{2}}\left(2+\frac{d \ln F_{z}}{d \ln \Omega_{0}}\right)
$$

where $F_{z}$ is the normal force exerted on the bottom plate. Thus, by plotting both the torque and the normal force versus the angular speed on a double logarithmic scale, the dependencies of the slopes $\frac{d \ln T}{d \ln \Omega_{0}}$ and $\frac{d \ln F_{z}}{d \ln \Omega_{0}}$ on the rim shear rate can be determined. This procedure yields $\sigma_{\theta z}$ and $N_{1}-N_{2}$. The apparent (or Newtonian) shear stress obtained with the parallel plate rheometric setup is:

$$
\sigma_{a}=\frac{2 T}{\pi R^{3}}
$$

\section{Novel trends in shear rheology}

\subsection{Fundamentals of extensional rheology}

A significant number of modern polymer processing operations which include (but are not limited to) melt blowing, fibre spinning, compression moulding, extrusion involve flows that combine both shear and extension. 
As compared to the case of shear flows, the material elements undergo much higher strains during extensional flows which typically leads to a strongly nonlinear dependence of the extensional stresses on the strain. The knowledge of the rheological behaviour in shear does not suffice to describe the behaviour during extension. The rheological response of a complex fluid undergoing shear such as a polymer solution or melt injected into a die or mould, drawn through an extrusion die, blow moulded, calendared etc. will not correctly predict the processing behaviour during such operations: inferring the extensional flow behaviour from rheological tests performed in shear is usually impossible.

Despite a clear need for extensional measurements of complex fluids, the development of instrumentation evolved slower than the development of shear rheometry. A first daunting task in this context is the generation of a spatially homogeneous extensional flow field. To generate an extensional motion in a fluid, one needs to bring the fluid in contact with a moving solid surface. This generally creates shear in the vicinity of the solid surface which alters the kinematics of the extensional flow. A second difficulty relates to the need of generating high strains during extension. This requires the development of motion control systems able to operate within a broad range of speeds and achieve significant travel distances.

Unlike in the case of shear rheometry, the design and instrumentation of an extensional rheometric device depend on the range of extensional viscosities to be measured. After providing the reader with a basic description of the kinematics of extensional flows, we will describe several such extensional rheometric devices highlighting both their advantages and practical limitations.

Kinematics of extensional flows A pure extensional flow is an irrotational flow which lacks both vorticity and shear, Bird et al. (1977). As compared to a laminar shear flow where neighbouring fluid elements separate linearly with time, in an extensional flow the separation is exponential in time. Consequently, such flows are very efficient in elongating and orienting the microscopic structural units of a complex fluid which makes them a valuable of probing the microstructure of a complex fluids. A simple extensional flow may be described by the following velocity field:

$$
\begin{array}{r}
v_{x}=-\frac{1}{2}(1+b) \dot{\epsilon} x \\
v_{y}=-\frac{1}{2}(1-b) \dot{\epsilon} y \\
v_{z}=\dot{\epsilon} z
\end{array}
$$




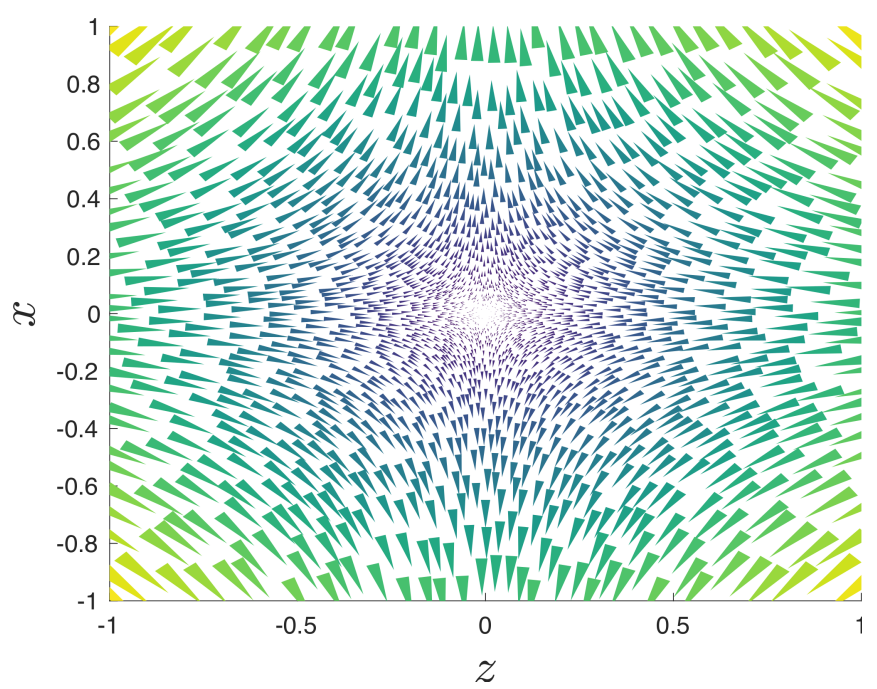

Figure 5. Steady elongational flow field $(b=0)$.

where $b$ is a constant that affects the way the streamlines change with rotation about the $\mathrm{z}$ axis and $\dot{\epsilon}$ is the rate of deformation of fluid elements. Three distinct types of extensional flows may be obtained for different choices of the parameter $b$ and of the sign of the rate of deformation:

1. $b=0, \dot{\epsilon}>0$ - uniaxial extension

2. $b=0, \dot{\epsilon}<0$ - biaxial stretching

3. $b=1$ - planar elongation

A typical extensional flow field obtained for $b=0$ is illustrated in Fig. 5 .

The deformation of a cubic fluid element of unitary volume by each of the three types of extensional flows is schematically illustrated in Fig. 6.

In practical applications even in the case when the deformation field is steady, the response of the material is usually unsteady meaning that the material reaches a steady dynamic regime only after a finite period of time. This is because fluids are characterised by a finite response time. In the case of Newtonian fluid, the characteristic time scale of the response is the viscous time whereas for non-Newtonian fluids is a relaxation time which describes how fast the micro-structure "emph" to the externally applied deformation field. Bearing this in mind, it appears natural that the material functions characterising the response to an extensional flow should be sought as time dependent (i.e. "transient") even in the case when the deformation field is 


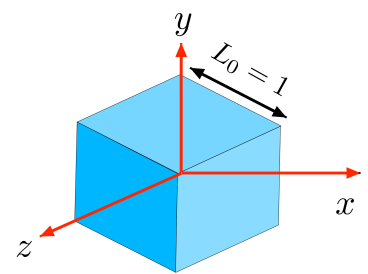

(a)

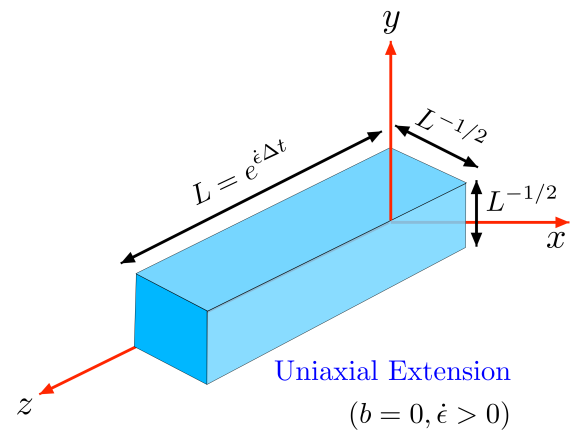

(b)

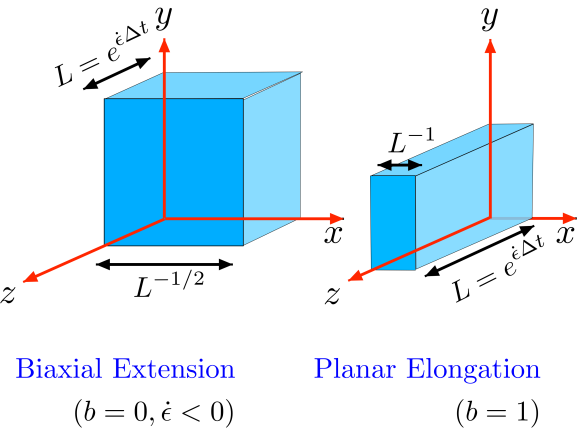

(c)

Figure 6. Deformation of a cube of unitary volume (a) during the time interval $\Delta t$ by three types of shear free flow: (b) uniaxial extension $(b=0$, $\dot{\epsilon}>0)$, (c) biaxial extension $(b=0, \dot{\epsilon}<0)$, (d) planar elogation $(b=1)$.

steady $\left(\frac{d \dot{\epsilon}}{d t}=0\right)$. Thus, the transient extensional viscosities may be defined as:

$$
\begin{aligned}
\eta_{1}^{+}(\dot{\epsilon}, t) & =\frac{\sigma_{z z}-\sigma_{x x}}{\dot{\epsilon}} \\
\eta_{2}^{+}(\dot{\epsilon}, t) & =\frac{\sigma_{x x}-\sigma_{y y}}{\dot{\epsilon}}
\end{aligned}
$$

For the case of the uniaxial extension $(b=0) \sigma_{x x}=\sigma_{y y}$ and the response of the material to deformation is characterised by a single transient extensional viscosity, $\eta^{+}=\eta_{1}^{+}$.

The total strain accumulated by the material elements during the extensional process may be computed by direct integration of the equations of motion Eq. 41. In the case of the uniaxial extension $(b=0)$, the position of 
a material element labelled by [j] varies exponentially in time, $X^{j}=X_{0}^{j} e^{\dot{e} t}$ and the strain between two neighbouring material elements is the Hencky strain defined logarithmically by:

$$
\epsilon_{H}=\dot{\epsilon} t=\ln \left[\frac{\Delta X(t)}{\Delta X(0)}\right]
$$

For a linear viscoelastic material characterised by a discrete spectrum of relaxation times $\left\{\lambda_{k}\right\}_{k=1, N}$ the response during uniaxial extension can be solved analitically:

$$
\eta^{+}(t)=\sum_{k=1}^{N} 3 \eta_{k}\left[1-e^{-\frac{t}{\lambda_{k}}}\right]
$$

In the asymptotic limit of long relaxation times $t \gg \max \lambda_{k}$, the response of the material approaches a steady state $\eta_{S S}^{+}=\lim _{t \rightarrow \infty} \eta^{+}(t)=3 \eta_{0}$ where $\eta_{0}$ is the zero shear viscosity of the material. This limiting value of the response is called the "linear viscoelastic envelope" (LVE). The knowledge of the LVE is crucial for the validation of extensional viscosity measurements because in a linear range of deformation all transient extensional viscosity curves should asymptotically approach the LVE. The concept of LVE is equally useful in introducing the term of "strain hardening" which is observed for a broad class of polymeric systems in the form of a substantial increase (up to several orders of magnitude) of the transient elongational viscosity with respect to the LVE. The magnitude of the strain hardening effect is quantified by the Trouton ratio:

$$
\operatorname{Tr}(\dot{\epsilon}, t)=\frac{\eta^{+}(t)}{\eta_{0}}
$$

The strain hardening is a rather complex phenomenon which depends on both the molecular architecture of the polymeric systems (e.g. branched polymeric systems exhibit stronger strain hardening) and the rate of extension $\dot{\epsilon}$. Thus, the quantisation of this effect by measuring the Trouton ratio is a rather sensitive probe of the branched structure of molten polymeric systems.

Capillary breakup rheometry of low viscosity fluids The capillary breakup extensional rheometry is a simple and reliable technique for assessing the extensional rheological properties of low to moderate viscosity fluids. The technique was first described by Bazilevsky and coworkers, Bazilevsky et al. $(1997,1990)$ and later revisited in a number of subsequent papers, Bazilevsky et al. (1981, 2001, 1994). 


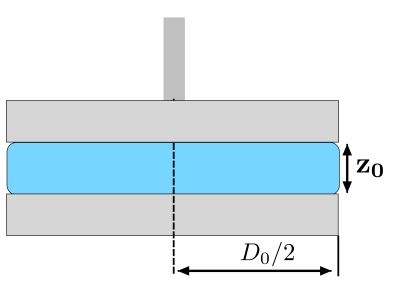

(a)

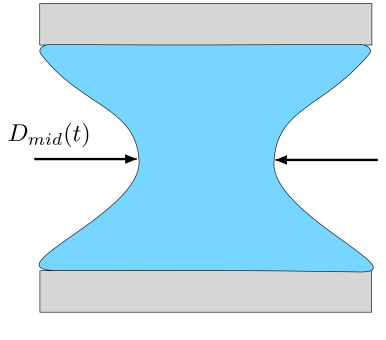

$(b)$

Figure 7. Schematic represenation of the capillary breakup device: (a) initial fluid meniscus. (b) fluid meniscus during the capillary thinning process.

In this technique, a drop of liquid is confined between two rigid plates initially set at a distance $z_{0}$ apart as schematically illustrated in Fig. 7(a). Next, by rapidly moving the top plate to a higher position, an axial stepstrain is imposed onto the liquid bridge Fig. 7(b). The shape of the liquid bridge evolves under the combined action of several physical processes: the capillary pressure which here plays the natural role of a "force transducer", viscous dissipation that the necking of the fluid filament and, in the case of polymeric fluids, the elastic forces that equally oppose the necking process.

Bazilevsky and coworkers were first to propose a theoretical framework to describe the thinning of a Newtonian and an Oldroyd-B fluid filament in terms of measurements of its radius, Bazilevsky et al. (1990). Their analysis has been extended by Entov and Hinch to account for both the effect of a spectrum of relaxation times and of the finite extensibility of the polymer chains, Entov and Hinch (1997).

For a Newtonian fluid of viscosity $\eta_{s}$ and surface tension coefficient $\gamma$ a local force balance together with the elimination of the pressure lead to the following equation for the mid-point diameter of the filament:

$$
3 \eta_{s}\left(-\frac{1}{D_{m i d}(t)} \frac{d D_{m i d}(t)}{d t}\right)=\frac{\gamma}{D_{m i d}(t)}
$$

The derivation of Eq. 46 assumed the total longitudinal stress along the fluid filament to be zero at all times. The term in the brackets may be understood as an extensional deformation rate of a Lagrangian fluid element at the mid-plane of the fluid column where the diameter $D_{\text {mid }}$ is 
measured. By integrating Eq. 46 a linear decay of the mid-point diameter of the filament with time is obtained:

$$
D_{\text {mid }}(t)=\frac{\gamma}{12 \eta_{s}}\left(t_{c}-t\right)
$$

Here $t_{c}=\frac{6 \eta_{s} D_{0}}{\sigma}$ is a critical time associated to the filament breakup and $D_{0}$ is the initial mid-point diameter of the filament. The Hencky $\epsilon_{H}(t)$ experienced by the fluid element at the mid-point of the liquid bridge at time $t$ can be defined using the mid-filament diameter:

$$
\epsilon_{H}(t)=2 \ln \left(\frac{D_{0}}{D_{\text {mid }}(t)}\right)
$$

The rate of extension at the mid-point of the filament may be readily obtained by differentiating Eq. 48:

$$
\dot{\epsilon}_{H}(t)=-\frac{2}{D_{\text {mid }}(t)} \frac{d D_{\text {mid }}(t)}{d t}
$$

From Eqs. 47 and 49 one can easily note that the rate of deformation is automatically set by the surface tension and the viscosity of the fluid.

Based on the measurements of the mid-point filament diameter, the apparent transient elongational viscosity may be estimated:

$$
\eta_{a p p}^{+}(t)=-\frac{\gamma}{\frac{d D_{m i d}(t)}{d t}}
$$

For viscoelastic fluids following the Oldroyd-B constitutive relationship and if the viscous dissipation may be neglected as compared to elastic and capillary terms in the force balance, an exponential decay of the mid-point diameter of the filament has been predicted theoretically, Bousfield et al. (1986); Bazilevsky et al. (1990); Renardy (1994); Entov and Hinch (1997):

$$
D_{\text {mid }}(t)=\left(\frac{\eta_{p} D_{0}^{4}}{2 \lambda \gamma}\right)^{1 / 3} e^{-\frac{t}{3 \lambda}}
$$

where $\eta_{p}=\eta-\eta_{s}$ is the polymer part of the viscosity. This relationship has been systematically verified experiementally, Anna and McKinley (2001).

Meissner rheometry of molten polymeric systems Meissner proposed a rotatory clamp extensional rheometer (RME) Meissner and Hostettler (1994) schematically illustrated in Fig. 8. The basic concept of the device was proposed at the end of $60^{\prime} s$ at the Research and Development department at the BASF, Ludwigshafen, Germany, Meissner (1969). 
The sample is clamped in between four counter-rotatory clamps. The sample was both heated and buoyed by gas injected from below. When the rotatory clamps were set in motion with a constant angular speed, the polymeric sample was experiencing a uniaxial extensional deformation. The samples were carefully prepared by extrusion and their initial length was rather large, $L_{0}=50 \mathrm{~mm}$. The large initial length of the sample might seem an unimportant detail but it is not. One of the particular concerns was related to the geometrical homogeneity of the sample which is of paramount importance in assuring a purely uniaxial deformation. This was specifically stated in page 20 of Ref. Meissner and Hostettler (1994): "When the elongation becomes inhomogeneous (for homogeneous samples the reason often is an inhomogeneous temperature field), any data processing becomes questionable". An initially long sample together with an extremely careful and elaborated mechanical design of the rotatory clamps insured a geometrical homogeneous deformation of the sample. The RME device could reach Hencky strains $\epsilon_{H}=7$ and could operate at strain rates of $\dot{\epsilon}_{H}=1 \mathrm{~s}^{-1}$. The transient tensile force $F(t)$ was measured by a force transducer was installed on the pairs of rotating clamps. For a geometrically homogeneous sample, the cross-sectional decays exponentially with time:

$$
A(t)=A_{0}\left(\frac{\rho_{S}}{\rho_{M}}\right)^{2 / 3} e^{-\epsilon_{H}}
$$

where $A_{0}$ is the initial cross sectional area measured in a solid state (at room temperature) and $\epsilon_{H}=\dot{\epsilon}_{H} t$ is the Hencky strain at time $t$. The pre-factor $\left(\frac{\rho_{S}}{\rho_{M}}\right)^{\frac{2}{3}}$ where $\rho_{S}$ is the density of the sample in a solid state while $\rho_{M}$ is its density in a molten accounts for the thermal expansion of the sample. The geometric homogeneity of the sample is crucial for the applicability of Eq. 52. In the case of a geometrically inhomogheneous sample the cross sectional area also depends on the position along the sample leading to a rate of deformation that is no longer equal to the nominal value $\dot{\epsilon}_{H}$ and depends on both the time and the position along the sample. As already stated above, Dr. Meissner was particularly keen about this aspect (which nowadays, unfortunately, no longer receives the deserved attention) and assessed the homogeneity of the sample during the extension by in-situ visualisation of the sample (see Fig. 19 in Ref. Meissner and Hostettler (1994)). The transient extensional viscosity may be computed according to:

$$
\mu^{+}(t)=\frac{F(t)}{A(t) \dot{\epsilon}_{H}}
$$




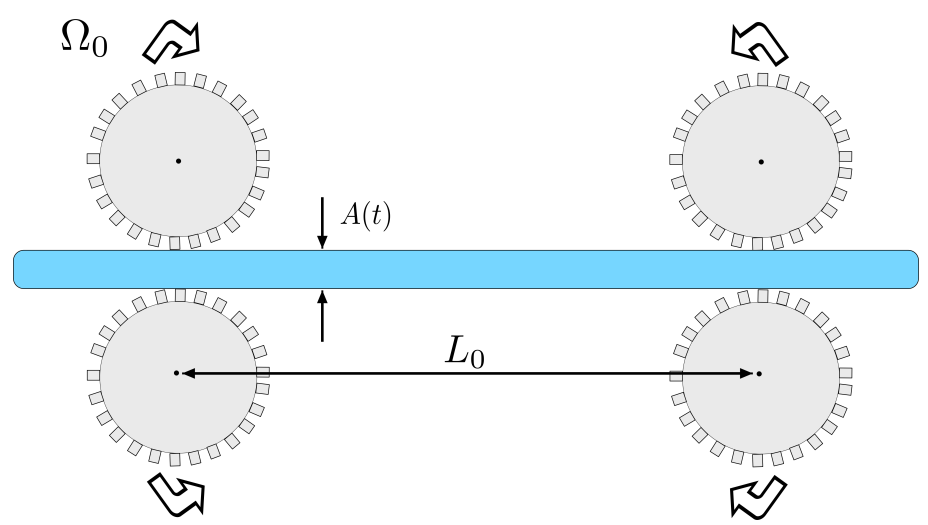

Figure 8. Schematic representation of the Meissner extensional rheometer.

Well ahead of its time, the RME device could operate in two distinct modes. The first one as described above is a "stressing mode" which corresponds to a constant rate of extension $\dot{\epsilon}_{H}$. Alternatively, however, the device could operate in "creep mode" which corresponds to a constant applied stress. Bearing in mind the limited technology available at the end of the $60^{\prime} s$, this was a remarkable achievement hard to implement even with todays technological advancements as it requires a specially developed controlled feedback system able to maintain the ratio $\frac{F(t)}{A(t) \dot{\epsilon}_{H}}$ constant during the extension.

Münstedt rheometry of molten polymeric systems Yet an another approach to the extensional rheology of molten polymeric system was proposed by H. Münstedt, Münstedt (1979). The Münstedt device is schematically illustrated in Fig. 9.

As opposed to the Meissner approach, the device proposed by Münstedt uses an initially short sample (S) with a cylindrical cross section and an initial diameter $D_{0}$ attached in a vertical position to the plates $P_{1}, P_{2}$ and immersed in an oil bath $O B$. The density of the oil is roughly equal to that of the sample thus minimising gravity and buoyancy effects which can cause inhomogeneous deformations of the specimen. While the bottom plate $P_{2}$ is fixed, the top plate $P_{1}$ is moved vertically by a ac-servo motor controlled by a personal computer. If $L_{0}$ is the initial length of the sample, $L(t)$ its length at time $t$ the nominal Hencky strain is defined:

$$
\epsilon_{H}=\ln \left(\frac{L(t)}{L_{0}}\right)
$$




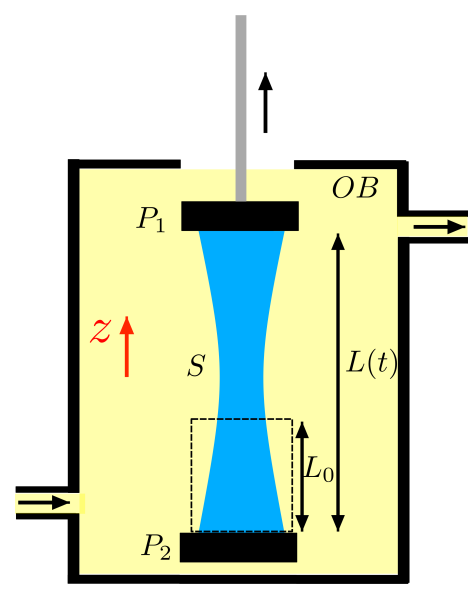

Figure 9. Schematic representation of the Münstedt extensional rheometer according to Ref. Münstedt (1979) (not in scale): (S) - sample, (OB) - oil bath, $\left(P_{1}\right)$ - top plate, $\left(P_{2}\right)$ - bottom plate.

The nominal rate of uniaxial deformation is:

$$
\dot{\epsilon}_{H}=\frac{L_{0}}{L(t)} \frac{d L(t)}{d t}
$$

The bottom plate $P_{2}$ is equipped with an accurate force transducer which measures the transient tensile force $F(t)$.

As the Meissner device, the Münstedt device may operate in two distinct modes, Münstedt and Laun (1979): "stressing mode" (at constant rate of deformation $\dot{\epsilon}$ ) and "creep" (at constant driving stress). In the stressing mode, a feedback loop insures an exponential increase of the distance between plates with time, $L(t)=L_{0} e^{\dot{\epsilon}_{H} t}$ and the transient elongational viscosity is computed as:

$$
\mu^{+}(t)=\frac{F(t)}{\dot{\epsilon}_{H} A(t)}
$$

Here $A(t)=\pi D^{2}(t) / 4$ is the transient cross-sectional area of the sample with $D(t)$ being the diameter of the sample at time $t$.

As in the case of the Meissner device, the homogeneity of the deformation states is crucial in reliably assessing the transient extensional viscosity of the material. It has been demonstrated experimentally that if this condition is not fulfilled, the Hencky strain is not uniform along the sample and the interpretation of the measurements becomes questionable, Burghelea et al. 
(2009); Starý et al. (2015); Burghelea et al. (2011, 2012). If the homogeneity condition is fulfilled, due to the incompressibility of the polymer melt, it can be readily shown that $D(t)=D_{0} e^{-\epsilon_{H} / 2}$ and Eq. 56 becomes:

$$
\mu^{+}(t)=\frac{4 F(t)}{\pi \dot{\epsilon}_{H} D_{0}^{2} e^{-\epsilon_{H}}}
$$

In a stressing operating mode, a different feedback loop maintains the ratio $\sigma^{+}(t)=F / A(t)=F L(t) /(V)$ constant where $V$ is the volume of the sample. Again, these measurements rely on the geometrical homogeneity of the sample and its incompressibility.

Filmament stretching rheometry of molten polymeric systems The filament stretching extensional rheometer (FISER) for viscous fluids was proposed by Tirtaatmadja and Sridhar, Tirtaatmadja and Sridhar (1993). The original design of the FISER is schematically illustrated in Fig. 10. Like the Münstedt device, the FISER uses a constant volume sample with a small initial length $L_{0}$ clamped in between two plates $P_{1}, P_{2}$ which move in opposite directions with equal speeds $u(t)$.

Variants of the device with the sample oriented vertically were later proposed, Anna et al. (2001); McKinley and Sridhar (2002); Bach et al. (2003, 2002). Unlike in the case of the Münstedt device, the sample is not immersed in an oil bath which, at low deformation rates, makes the measurements problematic due to gravity sagging effects, Anna et al. (2001). The theoretical background of the transient elongational viscosity measurements using the FISER device is given in Ref. Szabo (1997). Due to the lack of an oil bath the force balance includes a non-negligible surface tension term and, in a vertical configuration, a gravity term as well. In addition, depending on the operating speeds, an extra inertial term may be involved. Thus, the stress difference computed at the mid-point of the filament is:

$$
\sigma_{z z}(t)-\sigma_{r r}(t)=\frac{4 F(t)}{\pi D_{m i d}(t)^{2}}-\frac{2 \gamma}{D_{m i d}(t)}-\frac{\rho_{M} g L(t)}{2}-\frac{\rho_{M} \dot{\epsilon}_{H}(t)^{2} L(t)^{2}}{8}
$$

where $\gamma$ is the surface tension coefficient of the sample and $\rho_{M}$ its viscosity. The last term in the right-hand side of Eq. 58 which accounts for the inertia may be neglected if:

$$
\frac{\rho_{M} \dot{\epsilon}_{H} L}{8 \mu^{+}} \ll 1
$$

This inequality above is satisfied for highly viscous polymer melts but may break down for materials with smaller viscosities tested at high rates of deformation. The transient extensional viscosity may be calculated as: 


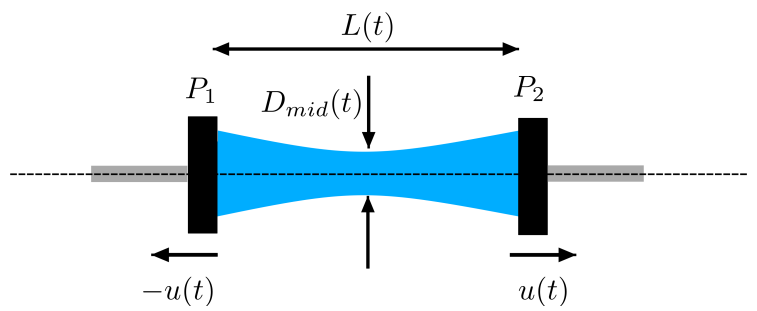

Figure 10. Schematic representation of the filament stretching rheometer according to Ref. Tirtaatmadja and Sridhar (1993) (not in scale).

$$
\begin{array}{r}
\mu^{+}(t)=\frac{\sigma_{z z}(t)-\sigma_{r r}(d)}{\dot{\epsilon}_{H}(t)}=\frac{4 F(t)}{\pi \dot{\epsilon}_{H}(t) D_{m i d}(t)^{2}}-\frac{2 \gamma}{\dot{\epsilon}_{H}(t) D_{\text {mid }}(t)} \\
-\frac{\rho_{M} g L(t)}{2 \dot{\epsilon}_{H}(t)}-\frac{\rho_{M} \dot{\epsilon}_{H}(t) L(t)^{2}}{8}
\end{array}
$$

The FISER device may be operated in two distinct ways. The first way is very similar to the operating mode of the Münstedt device. First, the Hencky strain is computed from the instantaneous length of the sample according to Eq. 55. If the deformation of the sample is geometrically homogeneous, the mid-point diameter of the sample may be computed as $D_{\text {mid }}(t)=D_{0} e^{-\epsilon_{H}(t) / 2}$. Finally, the transient elongational viscosity according to Eq. 60.

Alternatively, rather than monitoring the instantaneous length $L(t)$ of the sample in order to compute the Hencky strain, one could measure the instantaneous diameter of the sample $D_{\text {mid }}(t)$ measured at the midpoint of the sample. Such measurements may be performed either using a laser sheet and a photomultiplier or by imaging the sample with a digital camera. The local Hencky strain may be computed as for the CABER device (Eq. 48):

$$
\epsilon_{H}^{m i d}(t)=2 \ln \left(\frac{D_{0}}{D_{\text {mid }}(t)}\right)
$$

Consequently, the rate of deformation at the mid-point of the sample is:

$$
\dot{\epsilon}_{H}^{m i d}(t)=-\frac{2}{D(t)} \frac{d D(t)}{d t}
$$

If the deformation is geometrically inhomogeneous, the rate of deformation at the mid-point of the sample differs from the nominal value given by 
Eq. 55 and is not necessarily constant in time. This issue may be addressed by employing a special feedback loop which modifies in real time the separating speed of the plates based on the local measurements of the mid-point diameter of the filament in order to maintain the local rate of deformation given by Eq. 62 constant, Anna et al. (2001); Bach et al. (2003, 2002).

The FISER device has been recently become available commercially from Rheo Filament.

Sentmanat rheometry of molten polymeric systems An ingenious method of using a commercial rotational rheometer to perform measurements of the transient elongational viscosity was proposed by Martin Sentmanat, Sentmanat (2003a,b, 2004).

The idea consisted of designing a fixture that can be mounted on the shaft of a rotational rheometer and "convert" the rotation at a constant angular speed $\Omega_{0}$ in a uniaxial extension flow, Fig. 11. The Sentmanat extensional fixture consists of two identical drums of radius $R$ separated by a fixed distance $L_{0}$ inter-coupled by a system of gears (not shown in Fig. 11) such as the rotation of the cylinder connected to the shaft triggers the rotation of the second cylinder at a same angular speed but in an opposite sense. This counter-rotative motion of the cylinders will induce a uniaxial deformation of a rectangular shaped sample rigidly fixed by a system of clamps onto the frontal surface of the cylinders, Fig. 11(a).

For a constant angular speed of the shaft, the Hencky strain associated to the uniaxial extension of the sample increases linearly with time:

$$
\epsilon_{H}=\frac{2 \Omega_{0} R}{L_{0}} t
$$

Accordingly, the Hencky strain rate is given by:

$$
\dot{\epsilon}_{H}=\frac{2 \Omega_{0} R}{L_{0}}
$$

The resistance of the sample to the extensional deformation manifests through a transient tangential force $F(t)$ acting on each cylinder and related to the transient torque $T(t)$ acting on the shaft of the rheometer via:

$$
F(t)=\frac{T(t)}{2 R}
$$

If an affine deformation of the specimen is assumed, its cross sectional area $A(t)$ (see Fig. $11(\mathrm{~b})$ ) will decay exponentially with time according to: 


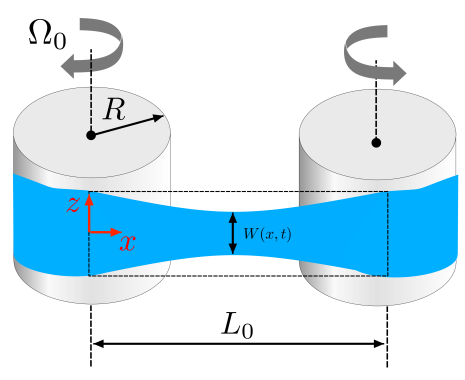

(a)

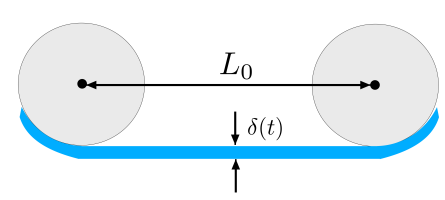

(b)

Figure 11. Schematic representation of the Sentmanat extensional fixture: (a) front view (b) top view.

$$
A(t)=A_{0}\left(\frac{\rho_{S}}{\rho_{M}}\right)^{\frac{2}{3}} e^{-\dot{\epsilon}_{H} t}
$$

where $A_{0}$ is the initial cross sectional area measured in a solid state (at room temperature). The pre-factor $\left(\frac{\rho_{S}}{\rho_{M}}\right)^{\frac{2}{3}}$ where $\rho_{S}$ is the density of the sample in a solid state while $\rho_{M}$ is its density in a molten accounts for the thermal expansion of the sample.

For a purely uni-axial extension at a constant Hencky strain rate, the transient elongational viscosity may be expressed:

$$
\eta^{+}(t)=\frac{F(t)}{\dot{\epsilon}_{h} A(t)}
$$

where the cross-sectional area $A(t)$ is given by Eq. 66 .

As compared to the other techniques of measuring the transient elongational visocosity of polymer melts, the Sentmanat has a number of advantages as well as some potential disadvantages. A first major advantage of this technique comes from its simplicity: the extensional fixture may be quickly coupled to any commercially available rotational rheometer and the sample can be quickly loaded. This is certainly not the case of the previously discussed methods which all require sophisticated machining, instrumentation and digital control systems as well as a more complicated loading procedure of the sample. Second, as compared to other techniques, the Sentmanat solution is rather affordable.

However, this technique equally has some drawbacks. First, the sample is held in air and, consequently, gravity sagging effects may bias the mea- 
surements of the transient elongational viscosity. These effects may become significant in a range of low Hencky strain rates. A second drawback comes from the lack of visual information on the evolution of the sample during extension: when used for molten polymeric systems, the extensional fixture is enclosed in an oven which makes the visualisation of the sample impossible. Thus, if some undesired effects such as gravity sagging or slippage of the sample at the contact points with the cylinders come into play, the operator will only see spurious data after the end of the experiment. A third and perhaps the most important drawback of this technique relates to the impossibility of assuring a geometrical uniform deformation of the sample at all times. In terms of flow kinematics, this means that the frontal shape of the sample must remain rectangular at all times during the extension (as hinted by the dotted lines in Fig. 11(a)). Any deviation from this would translate into a rate of deformation that differs from the nominal value given by Eq. 64 and is a function of the horizontal coordinate $x$. Equation 67 used to compute the transient extensional viscosity using the torque measurements performed by the rotational rheometer is applicable if and only if the deformation is a purely uniaxial one which, of course, is no longer the case if the shape of the sample deviates from a rectangular one.

This aspect is generally neglected while performing measurements with the Sentmanat fixture. On the other hand, according to the Considère rule, an inhomogeneous deformation will regularly appear at a finite Hencky strain, Considére (1885). This inhomegeneous deformation is triggered by the presence of the rigid boundary conditions at the clamping points of the sample and appears in the form of a primary necking.

\section{Bibliography}

Shelley L. Anna and Gareth H. McKinley. Elasto-capillary thinning and breakup of model elastic liquids. Journal of Rheology, 45(1):115-138, 2001. doi: 10.1122/1.1332389. URL https://doi.org/10.1122/1. 1332389 .

Shelley L. Anna, Gareth H. McKinley, Duc A. Nguyen, Tam Sridhar, Susan J. Muller, Jin Huang, and David F. James. An inter-laboratory comparison of measurements from filament-stretching rheometers using common test fluids. Journal of Rheology, 45(1):83-114, 2001. doi: 10.1122/1.1332388. URL https://doi.org/10.1122/1.1332388.

Anders Bach, Henrik Koblitz Rasmussen, Pierre-Yves Longin, and Ole Hassager. Growth of non-axisymmetric disturbances of the free surface in the filament stretching rheometer: experiments and simulation. Journal of Non-Newtonian Fluid Mechanics, 108(1):163 - 186, 
2002. ISSN 0377-0257. doi: https://doi.org/10.1016/S0377-0257(02) 00129-5. URL http://www.sciencedirect.com/science/article/ pii/S0377025702001295. Numerical Methods Workshop S.I.

Anders Bach, Henrik Koblitz Rasmussen, and Ole Hassager. Extensional viscosity for polymer melts measured in the filament stretching rheometer. Journal of Rheology, 47(2):429-441, 2003. doi: 10.1122/1.1545072. URL https://doi.org/10.1122/1.1545072.

A. V. Bazilevsky, S. I. Voronkov, V. M. Entov, and A. N. Rozhkov. Orientation effects in the breakup of jets and filaments of dilute polymer solutions. Dokl. Akad. Nauk SSSR, 2:336-339, 1981.

A. V. Bazilevsky, V. M. Entov, and A. N. Rozhkov. Liquid filament microrheometer and some of its applications. Proceedings of the 3rd European Rheology Conference, pages 865-929, 1990.

A. V. Bazilevsky, A. N. Rozhkov, and A. Stavisky. Proceedings of the Fourth European Rheology Conference, pages 468-470, 1994.

A. V. Bazilevsky, Lerner M. M. Entov, V. M., and A. N. Rozhkov. Failure of polymer solution filaments. Polym. Sci. Ser A, 39:716-726, 1997.

A. V. Bazilevsky, V. M. Entov, and A. N. Rozhkov. Breakup of an oldroyd liquid bridge as a method for testing the rheological properties of polymer solutions. Polym. Sci. Ser A, 43:716-726, 2001.

E. C. Bingham. The history of the society of rheology from 1924-1944. unpublished,Executed By Directive From The Society, Original archived in the Niels Bohr Library and Archives, American Institute of Physics, College Park, MD, cca 1945.

R.B. Bird, O. Hassager, R.C. Armstrong, and C.F. Curtiss. Dynamics of Polymeric Liquids, vol. 1. Wiley, New York, 1977.

D.W. Bousfield, R. Keunings, G. Marrucci, and M.M. Denn. Nonlinear analysis of the surface tension driven breakup of viscoelastic filaments. Journal of Non-Newtonian Fluid Mechanics, 21(1):79 - 97, 1986. ISSN 0377-0257. doi: https://doi.org/10.1016/0377-0257(86) 80064-7. URL http://www.sciencedirect.com/science/article/ pii/0377025786800647.

Teodor I. Burghelea, Zdeněk Starý, and Helmut Münstedt. Local versus integral measurements of the extensional viscosity of polymer melts. Journal of Rheology, 53(6):1363-1377, 2009. doi: 10.1122/1.3237024. URL http://dx.doi.org/10.1122/1.3237024.

Teodor I. Burghelea, Zdeněk Starý, and Helmut Münstedt. On the "viscosity overshoot" during the uniaxial extension of a low density polyethylene. Journal of Non-Newtonian Fluid Mechanics, 166(19):1198 - 1209, 2011. ISSN 0377-0257. doi: https://doi.org/10.1016/j.jnnfm.2011.07. 007. URL http://www.sciencedirect.com/science/article/pii/ S0377025711001819. 
Teodor I. Burghelea, Zdenek Stary, and Helmut Munstedt. Response to the "reply to: "on the "viscosity overshoot" during the uniaxial extension of a low density polyethylene' by rasmussen et al.". Journal of Non-Newtonian Fluid Mechanics, 171-172:107 - 108, 2012. ISSN 03770257. doi: https://doi.org/10.1016/j.jnnfm.2012.01.005. URL http:// www.sciencedirect.com/science/article/pii/S0377025712000067.

A. Considére. Memoire sur l'emploi du fer et de l'acier dans les constructions. Annales des Ponts et Chaussées, 6(9):574-775, 1885.

M Couette. Sur un nouvel appareil pour l'étude du frottement des fluides. Compt. Rend. Acad. Sci. Paris, 107:388-390, 1880.

V.M. Entov and E.J. Hinch. Effect of a spectrum of relaxation times on the capillary thinning of a filament of elastic liquid. Journal of NonNewtonian Fluid Mechanics, 72(1):31 - 53, 1997. ISSN 0377-0257. doi: https://doi.org/10.1016/S0377-0257(97)00022-0. URL http:// wWw. sciencedirect.com/science/article/pii/S0377025797000220.

C. W. Macosko. Rheology: principles, measurements, and applications. Wiley, New York, 1994. ISBN 0471185752.

Gareth H. McKinley and Tamarapu Sridhar. Filament-stretching rheometry of complex fluids. Annual Review of Fluid Mechanics, 34(1):375-415, 2002. doi: 10.1146/annurev.fluid.34.083001.125207. URL https://doi. org/10.1146/annurev.fluid.34.083001.125207.

J. Meissner. Rheometer zur untersuchung der deformations-mechanischen eigenschaften yon kunststoff- schmelzen unter definierter zugbeanspruchung. Rheologica Acta, pages 78-88, 1969.

J. Meissner and J. Hostettler. A new elongational rheometer for polymer melts and other highly viscous liquids. Rheologica Acta, 33:1-21, 1994.

H. Münstedt. New universal extensional rheometer for polymer melts. measurements on a polystyrene sample. Journal of Rheology, 23(4):421436, 1979. doi: 10.1122/1.549544. URL https://doi.org/10.1122/1. 549544 .

Helmuth Münstedt and H. M. Laun. Elongational behaviour of a low density polyethylene melt ii. Transient behavior in constant stretching rate and tensile creep experiments. Comparison with shear data. Temperature dependence of the elongational properties. Rheologica Acta, 18:492-504, 1979.

Michael Renardy. Some comments on the surface-tension driven break-up (or the lack of it) of viscoelastic jets. Journal of Non-Newtonian Fluid Mechanics, 51(1):97 - 107, 1994. ISSN 0377-0257. doi: https://doi. org/10.1016/0377-0257(94)85005-4. URL http://www. sciencedirect. com/science/article/pii/0377025794850054.

M. Sentmanat. A novel device for characterizing polymer flows in uniaxial extension. ANTEC 03, Soc. Plastics Engineers, Tech. Papers, 49, $2003 \mathrm{a}$. 
M. Sentmanat. Dual windup extensional rheometer. U.S. Patent No. $6,578,413,2003 \mathrm{~b}$.

Martin L. Sentmanat. Miniature universal testing platform: from extensional melt rheology to solid-state deformation behavior. Rheologica Acta, 43(6):657-669, Dec 2004. ISSN 1435-1528. doi: 10.1007/s00397-004-0405-4. URL https://doi.org/10.1007/ s00397-004-0405-4.

Zdeněk Starý, Magdalena Papp, and Teodor Burghelea. Deformation regimes, failure and rupture of a low density polyethylene (ldpe) melt undergoing uniaxial extension. Journal of Non-Newtonian Fluid Mechan$i c s, 219: 35$ - 49, 2015. ISSN 0377-0257. doi: http://dx.doi.org/10.1016/ j.jnnfm.2015.02.007. URL http://www.sciencedirect.com/science/ article/pii/S0377025715000427.

Peter Szabo. Transient filament stretching rheometer. Rheologica Acta, 36(3):277-284, May 1997. ISSN 1435-1528. doi: 10.1007/BF00366669. URL https://doi.org/10.1007/BF00366669.

V. Tirtaatmadja and T. Sridhar. A filament stretching device for measurement of extensional viscosity. Journal of Rheology, 37(6):10811102, 1993. doi: 10.1122/1.550372. URL https://doi.org/10.1122/ 1.550372 . 